\title{
Sleep Disorders and Cognitive Impairment in Peritoneal Dialysis: A Multicenter Prospective Cohort Study
}

\author{
Youlu Zhao a-d Yuhui Zhang ${ }^{\text {a-d }} \quad$ Zhikai Yang $^{\text {a-d }}$ Jinwei Wang ${ }^{\text {a-d }}$ \\ Zuying Xionge Jinlan Liao ${ }^{\mathrm{e}}$ Li Hao $^{f}$ Guiling Liu ${ }^{\mathrm{f}}$ Yeping Ren ${ }^{\mathrm{g}}$ \\ Qin Wang ${ }^{g}$ Liping Duan $^{\mathrm{h}}$ Zhaoxia Zheng $^{\mathrm{h}}$ Jie Dong ${ }^{\mathrm{a}-\mathrm{d}}$ \\ ${ }^{a}$ Renal Division, Department of Medicine, Peking University First Hospital, Beijing, China; \\ ${ }^{b}$ Institute of Nephrology, Peking University, Beijing, China; ${ }^{C}$ Key Laboratory of Renal \\ Disease, Ministry of Health, Beijing, China; ${ }^{d}$ Key Laboratory of Renal Disease, Ministry of \\ Education, Beijing, China; ${ }^{e}$ Renal Division, Peking University Shenzhen Hospital, Shenzhen, \\ China; ${ }^{f}$ Renal Division, the Second Hospital of Anhui Medical University, Anhui, China; \\ ${ }^{g}$ Renal Division, the Second Affiliated Hospital of Harbin Medical University, Heilongjiang, \\ China; henal Division, Handan Central Hospital, Hebei, China
}

\section{Keywords}

Peritoneal dialysis · Sleep disorders - Cognitive impairment - End-stage renal disease ·

Chronic renal failure

\begin{abstract}
Background: Patients with chronic kidney disease experience a high burden of sleep disorders, and there are associations between sleep disorders and cognitive impairment. Objectives: Based on our previous cross-sectional survey on cognitive impairment in peritoneal dialysis, we further explored the relationship between sleep disorders and cognitive impairment, and predictors for declining cognitive function. Method: We conducted a multicenter prospective cohort study enrolling 458 clinically stable patients on peritoneal dialysis who were then followed up for 2 years. Demographic data, comorbidities, depression, and biochemistry data were collected at baseline. Sleep disorders including insomnia, restless legs syndrome, sleep apnea syndrome, excessive daytime sleepiness, possible narcolepsy, sleep walking and nightmares, and possible rapid eye movement behavior disorders were assessed using a panel of specific sleep questionnaires at baseline and in a second survey. Global cognitive function was measured at baseline and in a second survey, using the Modified Mini-
\end{abstract}

Youlu Zhao and Yuhui Zhang contributed equally to this work. 


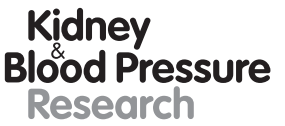

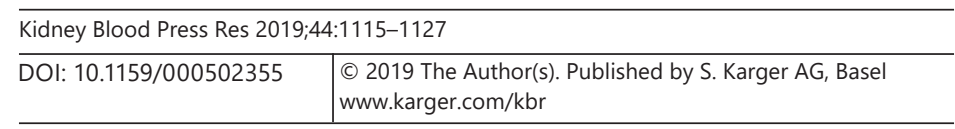

Zhao et al.: Cognitive Decline in Peritoneal Dialysis

Mental State Examination. Specific cognitive domains were evaluated using Trail-Making Test Forms A and B for executive function, and subtests of the Battery for the Assessment of Neuropsychological Status were used to asses immediate and delayed memory, visuospatial skills, and language ability. Results: Sleep disorders were common among peritoneal dialysis patients. The prevalence of cognitive impairment evaluated by the Modified Mini-Mental State Examination (3MS) increased from 19.8 to 23.9\%. Possible narcolepsy was associated with decreased Modified Mini-Mental State Examination scores at baseline. During follow-up, sleepwalking and nightmares were associated with higher risks of declined delayed memory in the longitudinal study. Conclusions: Possible narcolepsy was associated with general cognitive dysfunction, and sleep walking and nightmares were risk factors for impaired delayed memory.

(C) 2019 The Author(s)

Published by S. Karger AG, Basel

\section{Introduction}

Patients with chronic kidney disease (CKD) experience a high burden of sleep disorders caused by disease, treatment-related factors, and psychological factors. The prevalence of sleep disorders ranges from 20 to $70 \%$ among patients with CKD and from 44 to $95 \%$ among those undergoing dialysis [1-3]. Sleep disorders influence patients with CKD or maintaining dialysis in terms of mortality, quality of life, psychological status, and other aspects [4].

Evidence shows that there are associations between sleep disorders and cognitive impairment among patients with CKD and those on hemodialysis (HD). A cross-sectional study discovered that sleep-disordered breathing is associated with cognitive impairment, especially impaired verbal memory, in advanced CKD patients [5]. The other prospective cohort study indicated that patients undergoing HD with subjective sleep difficulties reported perceptual and memory problems [6]. It is noteworthy that dialysis patients have been found to suffer from cognitive impairment with an incidence of $27-67 \%[7,8]$. Cognitive impairment has also been shown to be an independent predictor of mortality and technique survival in this population. The use of peritoneal dialysis (PD) especially depends on normal cognitive functioning as it is a home care therapy requiring patients to self-monitor and self-manage their treatment.

Therefore, we aimed to investigate the associations between sleep disorders and general and specific cognitive function through a multicenter longitudinal PD cohort study.

\section{Materials and Methods}

\section{Study Design and Participants}

This was a multicenter prospective cohort-study. Five PD centers from 5 provinces participated. Data from each center were collected within a strict quality control framework. Demographic characteristics, comorbidities, biochemistry, along with assessments of cognitive function, depression, and sleep disorders, were collected at baseline. All of the participants were followed prospectively. We repeatedly measured the biochemical data, cognitive function, depression status, and sleep quality of patients who were still on PD between March 2015 and November 2015.

The PD patients were enrolled between March 2013 and November 2013. The inclusion criteria were: age $\geq 18$ years, having been undergoing PD for 3 months or longer and being clinically stable, and ability to complete all measurements and questionnaires as required. 
Patients were excluded if they had a systemic infection, acute cardiovascular events, active hepatitis or cancer, surgery or trauma in the month prior to the study, or any other studyobstructive condition such as severe eyesight loss, language incompatibility, illiteracy, a mental disturbance (preexisting dementia or confusion and various mental disorders), and upper limb disability. All of the participants received conventional glucose-based, lactatebuffered PD solutions (Ultrabag; Baxter Healthcare, Guangzhou, China).

\section{Clinical Characteristics}

Demographics and comorbidities, including age, gender, educational level, durations of PD, body mass index (BMI), systolic and diastolic blood pressure, primary kidney disease, presence of diabetes (DM), and history of cardiovascular disease (CVD), were recorded. CVD was recorded if one of the following conditions was present: angina, class III-IV congestive heart failure (New York Heart Association), transient ischemic attack, history of myocardial infarction or cerebrovascular accident, and peripheral arterial disease [9].

\section{Laboratory Methods}

The biochemical data included serum sodium, serum albumin, calcium and phosphate, triglycerides, total cholesterol, high-sensitivity C-reactive protein (hs-CRP), and hemoglobin, which were calculated as the mean of measurements taken over the preceding 3 months. Biochemical profiles were investigated using an automatic Hitachi chemistry analyzer.

\section{Sleep Assessment}

Assessments of cognitive function, depression status, and sleep disorders were performed in a separate room by trained medical staff in the morning. The sleep quality questionnaire included six parts to evaluate six major categories of sleep disorders. The insomnia part [10] covers nighttime symptoms of insomnia, daytime consequences of disturbed sleep, and frequency. The restless legs syndrome questionnaire includes International Restless Legs Syndrome Study Group (IRLSSG) criteria [11] and the IRLSSG rating scale [12], where a higher score in total identifies a much heavier disease burden. Excessive daytime sleepiness was assessed using the Epworth Sleepiness Scale (ESS) [13], with scores $\geq 10$ indicating the presence of the disease (the higher the value, the larger the tendency to experience excessive sleepiness). The validity and reliability of the Chinese version of the ESS had also been evaluated in PD patients [14]. Possible narcolepsy was screened for using the International Classification of Sleep Disorders (ICSD) questionnaire [15]. Sleepwalking and nightmares were assessed by Hatoum's nighttime disturbance questions [16]. The part on possible rapid eye movement behavior disorders consists of reporting symptoms of movement of the body or limbs or the presence of potential harmful aggressive behaviors related to dreams [17].

\section{Cognitive Function and Depression Status}

The overall cognitive function was assessed using the Modified Mini-Mental State Examination (3MS) [18]. Global cognitive impairment was defined as a score of $<80$ on the 3MS based on previous observational studies of cognitive function [19]. Because the mean scores on the 3MS vary by education, we used a 3MS cut-off point of $<75$ for individuals with less than a high school education and a 3 MS cut-off point of $<80$ for individuals with a high school education [20].

Specific cognitive functions were measured as executive function, immediate memory, delayed memory, visuospatial skill, and language ability. Trail-Making Tests A and B [21] were used to test executive function including decision-making and processing speed. Executive dysfunction was defined as a Trail-Making Test A duration of $>75 \mathrm{~s}$ and a Trail-Making Test B duration of $>180 \mathrm{~s}$ [22]. Subtests of the Repeatable Battery for the Assessment of 


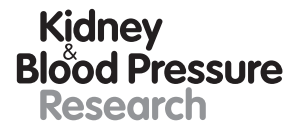

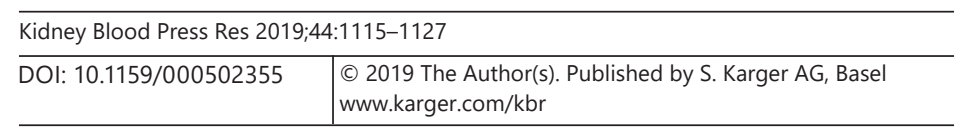

Zhao et al.: Cognitive Decline in Peritoneal Dialysis

Neuropsychological Status (RBANS) were adopted to assess immediate memory (list learning and story memory), delayed memory (list recall, list recognition, story recall, and figure recall), visuospatial skill (figure copying), and language ability (picture naming and semantic fluency) [23]. The reliability and validity of the RBANS have already been proven in Chinese populations [20, 24]. Raw scores were transformed to age-standardized $\mathrm{T}$ scores for all subtests of the RBANS. T scores less than 1 SD below the published mean in an educationgrouped Chinese population on a subtest indicated impairment [25].

Depression status was assessed using Zung's Self-Rating Depression Scale [26], which has been validated in general Chinese population and for various medical illnesses.

\section{Statistical Analysis}

Continuous data are presented as means \pm SD, except for durations of PD, RRF (residual renal function), and high sensitivity $\mathrm{C}$ reactive protein (hs-CRP) levels, which are given as medians and interquartile range (IQR) due to a high skewness. Categorical variables are presented as frequencies and proportions. Student's $t$ test, the Mann-Whitney U test, and the $\chi^{2}$ test were used to compare differences in basic characteristics. A paired Student $t$ test and the Wilcoxon signed rank test were employed to compare cognitive function parameters between baseline and the 2-year follow-up.

Using linear regression models, we explored the association between sleep disorders and cognitive function at baseline. Both univariable and multivariable linear regression models were used. Factors reported to be associated with cognitive impairment in previous studies, including demographic data (age, gender, educational level, and BMI), comorbidity status (DM, CVD, and depression status), and laboratory parameters (serum albumin, sodium, and hs-CRP) were all included in the models as independent variables. HR and 95\% CI were calculated.

We then used multivariable linear regression models to further analyze risk factors for worsening general and specific cognitive function, respectively, in the longitudinal study. The change of score in each cognitive parameter was used as a dependent variable. The same recognized cofactors mentioned above were included in the models as independent variables. The analysis for each cognitive domain was adjusted for the baseline level of the corresponding cognitive parameter.

The level of significance for all statistical tests was set at 0.05 . All of the analyses were performed using SPSS for Windows, version 22.0 (SPSS Inc., Chicago, IL, USA).

\section{Results}

\section{Basic Characteristics and Follow-Up}

Of the 667 patients who were eligible for this study, 493 (73.9\%) gave consent to participate. Among the 493 patients, 458 (92.9\%) completed baseline cognitive and sleep testing. Demographic and laboratory data for our participants were in accordance with the general characteristics of the PD population in China, with mean values as follows: age, 51.6 years; PD duration, 25.1 months; BMI, 22.9; hemoglobin level, $104.9 \mathrm{~g} / \mathrm{L}$; and serum albumin level, 36.2 g/L. Of these patients, $53.1 \%$ were male, $14 \%$ applied assisted PD, $23.6 \%$ had DM, $21.0 \%$ had a history of CVD, and $52.4 \%$ had an education of high school diploma or higher (Table 1).

A total of 165 patients were excluded for various reasons during the follow-up period (Fig. 1). Therefore, the remaining 293 patients were assessed for cognitive function and sleep disorders repeatedly. There were no significant differences in general or specific cognitive function parameters between the excluded patients and the remaining patients. The DM prevalence and serum hs-CRP levels were significantly higher in the excluded group $(p=$ 
Table 1. Clinical characteristics at baseline

\begin{tabular}{|c|c|c|c|c|}
\hline Variables & Total & $\begin{array}{l}\text { With sleep } \\
\text { disorders }(n=278)\end{array}$ & $\begin{array}{l}\text { Without sleep } \\
\text { disorders }(n=148)\end{array}$ & $p$ \\
\hline Subjects & $458(100)$ & $278(65.3)$ & $148(34.75)$ & - \\
\hline Age, years & $51.6 \pm 14.2$ & $52.3 \pm 13.9$ & $50.8 \pm 14.5$ & 0.292 \\
\hline Males & $243(53.1)$ & $158(56.8)$ & $68(45.9)$ & $0.032 *$ \\
\hline PD duration, months & $25.1(11.1-49.0)$ & $26.8(10.6-50.9)$ & $24.2(11.2-46.3)$ & 0.872 \\
\hline Assisted PD & $64(14.0)$ & $39(29.8)$ & $13(16.5)$ & $0.030^{*}$ \\
\hline DM & $108(23.6)$ & $77(27.7)$ & $25(16.9)$ & $0.013^{*}$ \\
\hline CVD & $96(21.0)$ & $64(23.0)$ & $28(18.9)$ & 0.327 \\
\hline Depression status & $227(50.7)$ & $162(58.3)$ & $54(36.5)$ & $0.000^{* * *}$ \\
\hline Level of education (high school and above) & $240(52.4)$ & $148(53.2)$ & $75(50.7)$ & 0.614 \\
\hline BMI & $22.9 \pm 3.5$ & $22.9 \pm 3.3$ & $22.9 \pm 3.9$ & 0.947 \\
\hline Mean arterial pressure, $\mathrm{mm} \mathrm{Hg}$ & $100.1 \pm 12.9$ & $100.5 \pm 13.4$ & $99.2 \pm 12.4$ & 0.360 \\
\hline Hemoglobin, g/L & $104.9 \pm 17.8$ & $104.2 \pm 17.6$ & $105.6 \pm 17.8$ & 0.439 \\
\hline Serum albumin, $\mathrm{g} / \mathrm{L}$ & $36.2 \pm 5.6$ & $35.8 \pm 5.6$ & $36.5 \pm 5.1$ & 0.178 \\
\hline Triglycerides, mmol/L & $2.0 \pm 1.3$ & $2.0 \pm 1.3$ & $1.9 \pm 1.2$ & 0.826 \\
\hline Total cholesterol, mmol/L & $4.7 \pm 1.1$ & $4.7 \pm 1.1$ & $4.8 \pm 1.1$ & 0.305 \\
\hline Serum sodium, mmol/L & $139 \pm 7.0$ & $139.4 \pm 3.2$ & $139.0 \pm 2.7$ & 0.194 \\
\hline Calcium, $\mathrm{mmol} / \mathrm{L}$ & $2.3 \pm 0.2$ & $2.3 \pm 0.2$ & $2.3 \pm 0.2$ & 0.332 \\
\hline Phosphate, mmol/L & $1.7 \pm 0.4$ & $1.7 \pm 0.5$ & $1.6 \pm 0.4$ & 0.120 \\
\hline Hs-CRP, mg/L & $2.8(0.9-8.6)$ & $3.1(0.9-9.5)$ & $2.4(0.8-7.0)$ & 0.088 \\
\hline $\mathrm{RRF}, \mathrm{mL} / \mathrm{min}$ & $2.2(0.0-5.7)$ & $2.0(0.0-5.3)$ & $2.5(0.0-6.3)$ & 0.185 \\
\hline Total Kt/V & $1.9 \pm 0.4$ & $1.9 \pm 0.4$ & $2.0 \pm 0.4$ & $0.026^{*}$ \\
\hline Total Ccr, L/week/1.73 m² & $58.4 \pm 17.8$ & $57.2 \pm 16.6$ & $59.9 \pm 19.4$ & 0.130 \\
\hline
\end{tabular}

The total number of subjects is 458 . Values are presented as means \pm SD, medians (IQR), or numbers (\%). Ccr, creatinine clearance per week. ${ }^{*} p<0.05$ and ${ }^{* * *} p<0.001$ (comparison between baseline and 2 years later).

0.006 and $p=0.04$, respectively). No significant difference was found in other demographic data or biochemical data between the 2 groups (for details, see [27]; Table 1).

Sleep Disorders, Clinical Characteristics, and Cognitive Impairment at Baseline

In this PD data set, the prevalence of having at least one of the six assessed sleep disorders at baseline was $65.3 \%$ (Fig. 2b). As shown in Table 1, compared to patients without sleep disorders, patients with at least one of the six assessed sleep disorders tended to be male, be more reliant on assisted PD, and have diabetes and depression. They also tented to have lower levels of total urea clearance per week (Kt/V).

The scores of each sleep disorder at baseline are shown as means \pm SD (Fig. 2a). In this PD data set, among the assessed sleep disorders, the most common one was excessive daytime sleepiness (47.6\%), followed by insomnia (22.6\%), sleep walking and nightmares (19.1\%), restless legs syndrome (7.9\%), possible rapid eye movement behavior disorders (7.2\%), and possible narcolepsy (4.7\%) (Fig. 2b). As shown in Figure 2c, having one sleep disorder accounted for $48 \%$ of the sleep disturbance group, having any two combined sleep disorders accounted for $26 \%$, and suffering from any five combined sleep disorders accounted for $2 \%$.

\section{Sleep Disorders and Changes in Cognitive Function during Follow-Up}

At the second survey, general and specific cognitive function and sleep disorders were repeatedly assessed in 293 patients (Table 2). When comparing scores for each test, we observed a worse performance on the Modified Mini-Mental State Examination (3MS) and excessive daytime sleepiness. However, we observed a better performance at the 2-year follow-up on Trail-Making Tests A and B, the immediate memory test, and visuospatial skills. 
Fig. 1. Flow chart of this study.

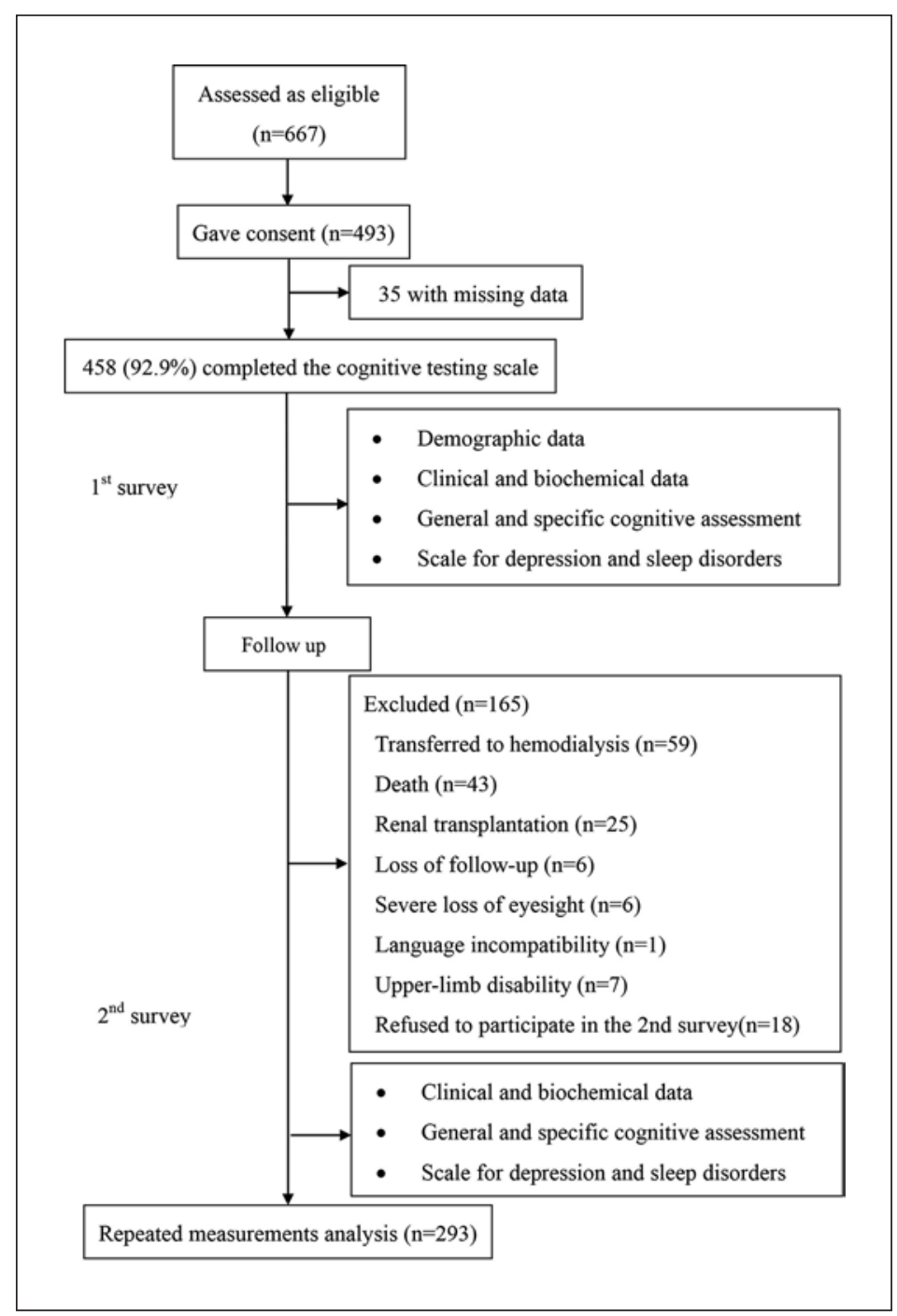

The scores for delayed memory and language skills, insomnia, restless legs syndrome, possible narcolepsy, sleep walking and nightmares, and possible rapid eye movement behavior disorders were not significantly different.

\section{Associations between Sleep Disorders and Cognitive Function at Baseline and during}

Follow-Up

Associations between sleep disorders and cognitive function at baseline are shown in Table 3. After adjusting for recognized confounders, possible narcolepsy was significantly associated with worse general cognition as assessed based on 3MS scores ( $p=0.002)$.

During the follow-up, the longitudinal relationship between sleep disorders and cognitive dysfunction was also explored. Using a multivariable linear regression model, sleepwalking and nightmares were found to be related to declining delayed memory ( $p=0.02$ and $p=0.044$, respectively). However, restless legs syndrome seemed to be associated with better executive function as assessed by both Trail-Making Test A and Trail-Making Test B. Beyond that, other sleep disorders were neither hazardous nor protective factors for the monitored sleep dysfunction (Table 4). 
A

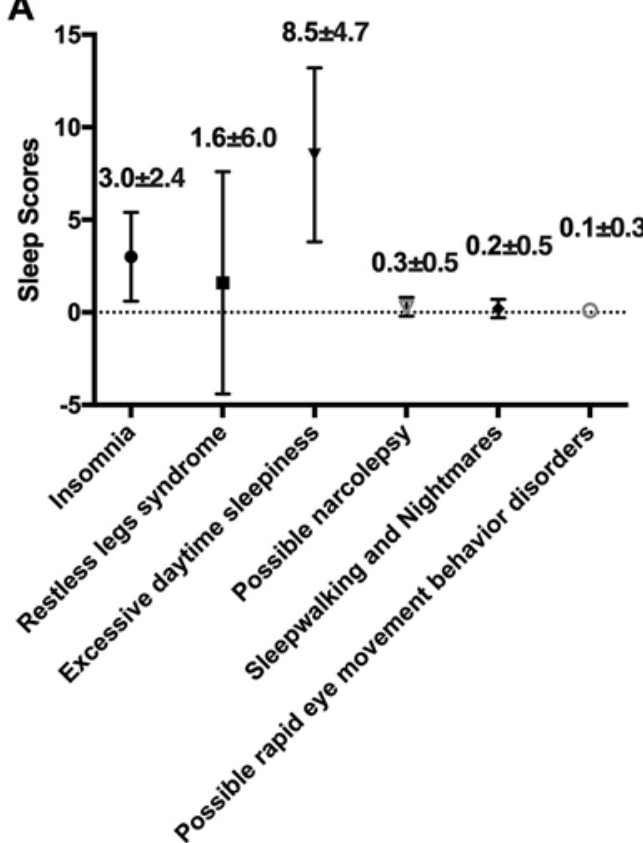

Sleep Categories
B

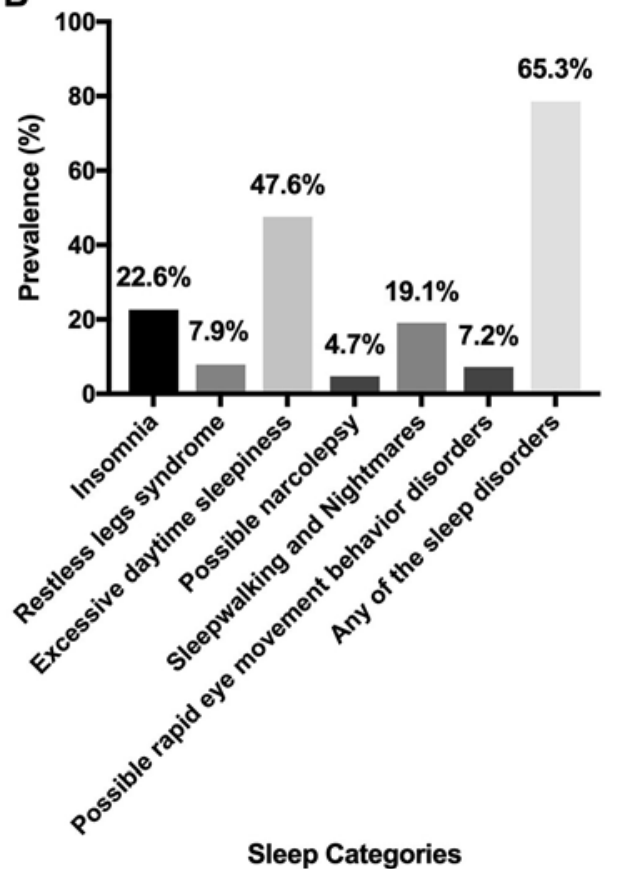

NA due to partial data missing

Any six combined

Any five combined

Any four combined

Any three combined

Any two combined

One sleep disorder

Fig. 2. Prevalence of sleep disorders.

\section{Discussion}

As far as we know, this is the largest multicenter prospective cohort study to measure the association between sleep disorders and cognitive impairment in patients undergoing PD. In this PD data set, the prevalence of having any of the 6 sleep disorders we assessed was $65.3 \%$. The most common specific disorder was excessive daytime sleepiness (47.6\%), followed by insomnia (22.6\%). At baseline, possible narcolepsy was associated with general cognitive dysfunction. Moreover, in the longitudinal analysis, sleepwalking and nightmares were risk factors for a decline in delayed memory capacity. 
Zhao et al.: Cognitive Decline in Peritoneal Dialysis

Table 2. Comparisons of cognitive function and sleep disorder parameters at the baseline assessment and the second survey

\begin{tabular}{|c|c|c|c|c|}
\hline & \multirow[t]{2}{*}{$\begin{array}{l}\text { All participants } \\
(n=458)\end{array}$} & \multicolumn{2}{|c|}{$\begin{array}{l}\text { Participants who completed the } \\
2 \text { assessments }(n=293)\end{array}$} & \multirow[t]{2}{*}{$p$} \\
\hline & & baseline & 2 years later & \\
\hline 3MS score & $84.7 \pm 12.3$ & $84.8 \pm 12.2$ & $83.1 \pm 14.8$ & $0.006^{* *}$ \\
\hline Cognitive impairment & 90 (19.7) & $58(19.8)$ & $70(23.9)$ & 0.161 \\
\hline Trail-Making Test A duration, s & $65.5(47.25-95)$ & $65(49-90)$ & $56.0(41.0-80.0)$ & $<0.001^{* * *}$ \\
\hline Trail-Making Test B duration, s & $150(106-233)$ & $144(103-227)$ & $144.0(102-193.5)$ & $0.025^{*}$ \\
\hline Immediate memory score & $74.0 \pm 17.9$ & $74.0 \pm 18.1$ & $76.6 \pm 18.4$ & $0.010^{*}$ \\
\hline Delayed memory score & $89.3 \pm 17.0$ & $89.3 \pm 17.7$ & $87.8 \pm 20.5$ & 0.155 \\
\hline Visuospatial skill score & $86.0 \pm 23.5$ & $86.7 \pm 23.7$ & $91.2 \pm 19.9$ & $0.004^{* *}$ \\
\hline Language ability score & $93.3 \pm 13.1$ & $93.5 \pm 13.7$ & $94.6 \pm 14.1$ & 0.207 \\
\hline Insomnia score & $3.0 \pm 2.4$ & $3.0 \pm 2.5$ & $3.3 \pm 2.4$ & 0.087 \\
\hline Restless legs syndrome score & $1.6 \pm 6.0$ & $1.6 \pm 5.8$ & $1.9 \pm 6.7$ & 0.416 \\
\hline Excessive daytime sleepiness score & $8.5 \pm 4.7$ & $8.3 \pm 4.5$ & $9.6 \pm 5.5$ & $<0.001^{* * *}$ \\
\hline Possible narcolepsy & $21(4.7)$ & $10(3.4)$ & $16(5.5)$ & 0.189 \\
\hline Sleepwalking and nightmares & $85(19.1)$ & $56(19.6)$ & $49(16.9)$ & 0.610 \\
\hline $\begin{array}{l}\text { Possible rapid eye movement behavior } \\
\text { disorder }\end{array}$ & $32(7.2)$ & $20(6.8)$ & $26(8.9 \%)$ & 0.337 \\
\hline
\end{tabular}

Values are presented as means $\pm \mathrm{SD}$, medians $(\mathrm{IQR})$, or numbers $(\%){ }^{*} p<0.05,{ }^{* *} p<0.01$, and ${ }^{* * *} p<0.001$ (comparisons between baseline and 2 years later).

The prevalence of possible narcolepsy in our study was similar to the results of previous studies in HD cohorts (1.4-15.9\%) [17, 28, 29]; it was higher than in the general population $(0.03-0.06 \%)$ [30]. The higher rate of narcolepsy in patients with end stage renal disease (ESRD) might be due to a changed orexin level, an autoimmune disorder, infections, toxins, or psychological stress, among other factors [31]. Whether experiencing narcolepsy during bag change could increase the risk of PD-related complications is unknown in previous literature. According to our data, possible narcolepsy was significantly associated with general cognitive function. The research on the association between possible narcolepsy and cognition is relatively new, and, consequently, data concerning patients with ESRD or CKD are sparse. Past studies conducted in the general population have found that narcoleptics have deficits in attention and memory [30].

The underlying mechanism of this reno-cerebral syndrome remains unclear. One hypothesis is that a hypocretin deficiency could drive the medial prefrontal cortex (a cortical region involved in associative function and attention) dysfunction due to less excitatory action and a consequent deficit of attention and memory [32]. It is suggested that acute sleep deprivation [33] and chronic sleep disorder [34] increase the cerebral amyloid- $\beta$ level, yet a relatively short-term partial sleep deprivation increases the orexin concentration [35], which is a significant indicator of cognitive impairment. Sleep disorders could be associated with cognitive impairment in patients with CKD. Cognitive decline and sleep disturbances could be manifestations of brain dysfunction in CKD. There are numerous co-contributors to sleep disorders and cognitive impairment in the progression of CKD, such as anemia, uremia, protein energy wasting, neuropsychological aspects [36], secondary hyperparathyroidism [37], unstable hemodynamics [38], systemic inflammation, and a frequent presence of electrolyte disorders [39]. Behind these clinical associations, there are also common pathologic structure changes in the regional brain. Past studies have suggested that an injured hippocampus, small-vessel ischemic brain disease, and deep white matter demyelination could be 
Kidney
Blood Pressure

Research
Kidney Blood Press Res 2019;44:1115-1127 DOI: 10.1159/000502355

(c) 2019 The Author(s). Published by S. Karger AG, Basel

www.karger.com/kbr

Zhao et al.: Cognitive Decline in Peritoneal Dialysis

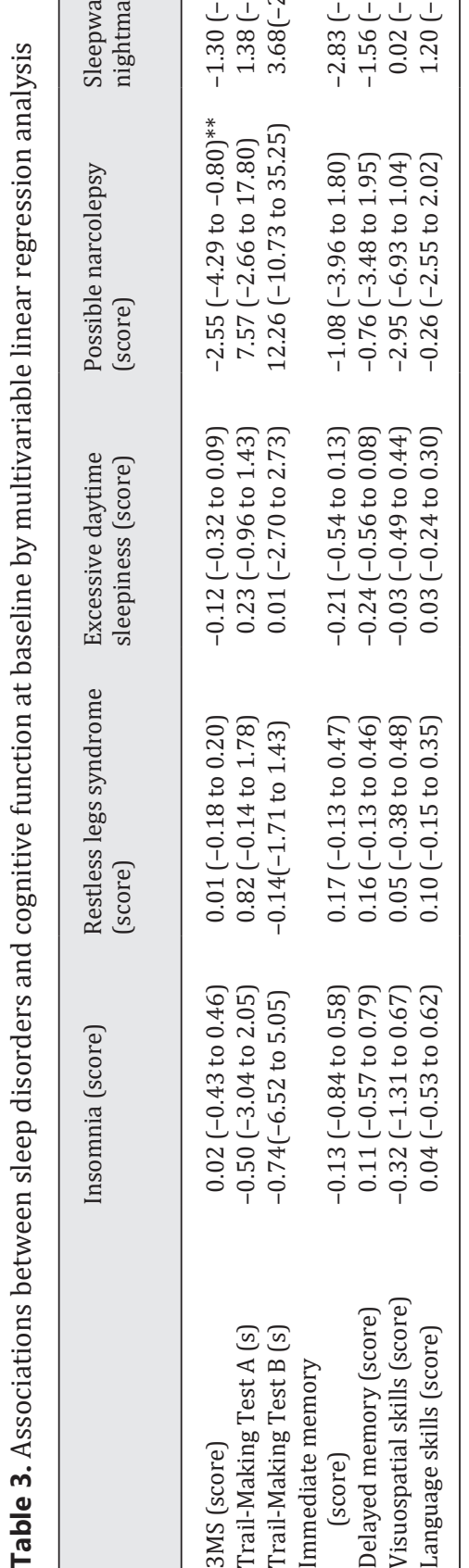

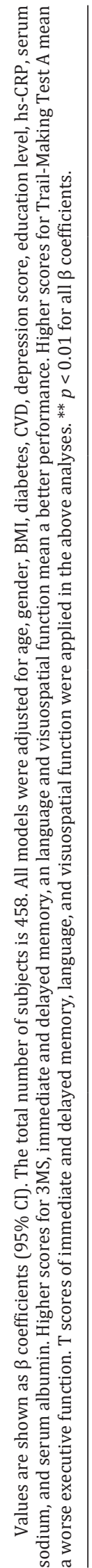

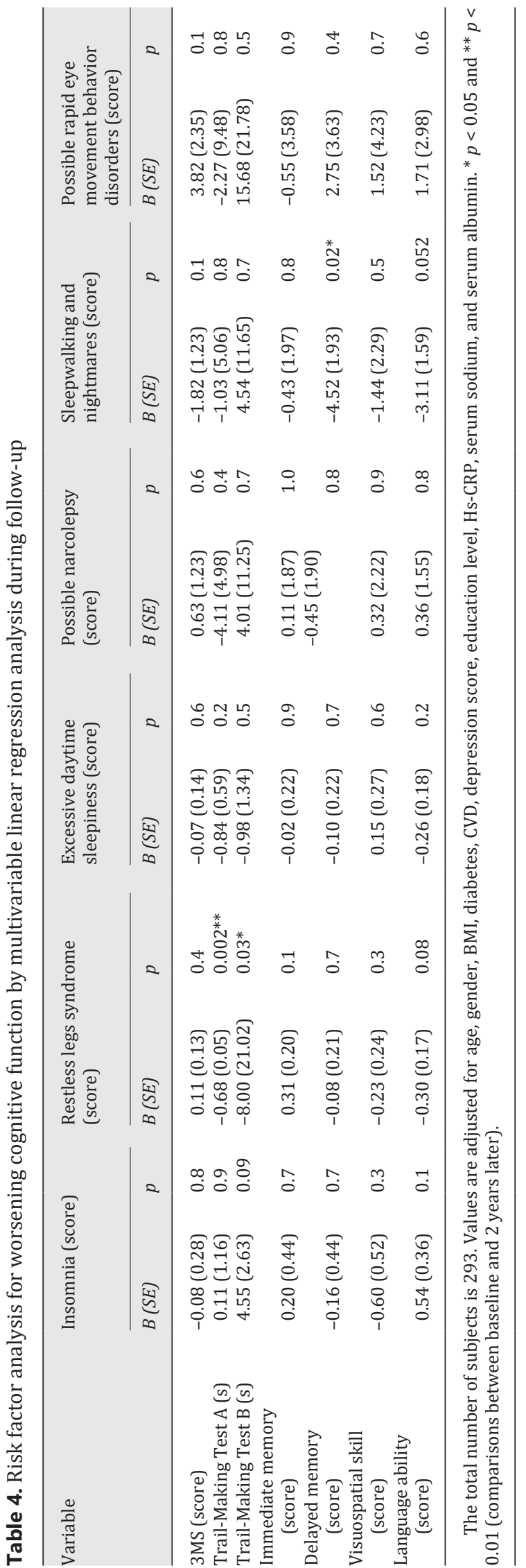




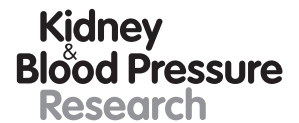

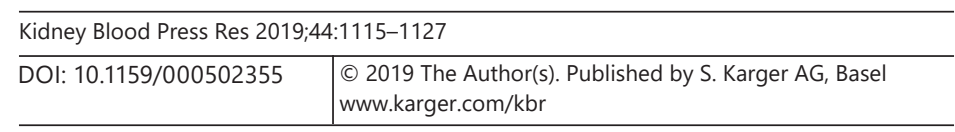

Zhao et al.: Cognitive Decline in Peritoneal Dialysis

seen in CKD patients with sleep disorders, which is vital for executive and memory function [39-42]. As narcolepsy is a severely disabling disorder, we encourage PD patients with potential symptoms to be assisted with bag exchange and exit site care in case of infectious and noninfectious complications. More research concerning this issue in a selected group would be urgent.

Sleepwalking and nightmares were found to be risk factors for a decline in delayed memory in our study. A review of the literature found no current evidence of this in a renalcompromised population; however, a correlation between global cognitive function and vivid dreams and nocturnal restlessness has been observed in patients with Parkinson disease [43]. The mechanisms underlying these associations remain to be determined; one hypothesis is that dysfunctions in the cholinergic systems and their projections to subcortical and cortical regions might play a role [44].

Our longitudinal study indicated that restless legs syndrome might be associated with a better executive function, which is against previous data revealing that executive functions are decreased in individuals with restless legs syndrome [45]. The potential mechanisms are not clear. Of note, there was a general improvement in executive function in the whole cohort, which is partly related to the amelioration of uremic symptoms during longterm dialysis treatment, as supported by a past study [46]. Also, we need to collect more information on nutrition, dialysis adequacy, and physical activity among patients with restless legs syndrome in a future study. The exploration of the association between restless legs syndrome and executive function should consider more potential confounders accordingly.

Several strengths of the present study are listed below. First, this study shows the association between sleep disorders and cognitive function in a large PD sample. Second, our cognitive measures included a broad and comprehensive range of tests encompassing a variety of cognitive domains, such as executive, memory, and language functions. Third, recognized confounders for cognitive impairment in general and dialysis populations were controlled in multivariable models.

We are also aware of limitations of this study. First, due to the nature of observational studies, it cannot be determined whether sleep disorders are pathogenic factors or solely risks factors for cognitive impairment. Second, almost $40 \%$ of the participants were not included in the second assessment, which could underestimate the trend in decreasing cognitive function as the drop-outs tended to be sicker at baseline. Besides, this study was performed exclusively in a Chinese population of patients who were treated with continuous ambulatory peritoneal dialysis (CAPD), and patients with severe cognitive impairment were excluded, thereby raising the possibility of selection/ascertainment bias. Moreover, polysomnography as a gold standard was not applied in this study. Since it is cumbersome, time consuming, expensive, and not applicable outside of the home environment, a panel of questionnaires consisting of multiple dimensions were measured. Most of the questionnaires have verified its validity and reliability in populations with CKD or under renal replacement therapy $[14,17,47,48]$.

In conclusion, we are the first study to demonstrate that sleep disorders and cognitive impairment have non-negligible associations in a PD population, as certain sleep disorders might cause specific domains of cognitive functioning to deteriorate. Moreover, possible narcolepsy, sleep walking, and nightmares were less commonly studied, although they had high potential risks especially among individuals undergoing PD. Therefore, more attention needs to be focused on this aspect so that more light can be shed on the pathophysiology, precaution, and comprehensive management pertaining to this field. 


\section{Acknowledgement}

The authors would like to express their appreciation to the patients, doctors, and nursing staff of the Peritoneal Dialysis Centers of Peking University First Hospital. The authors would also like to express their gratitude to Prof. Yingdong Zheng of the School of Public Health of Peking University for his dedication in the statistical analysis in the study.

\section{Statement of Ethics}

The ethics committee of Peking University First Hospital approved this study (approval No. 2013[587]). Patients gave written consent for their information to be stored in the hospital database and for it to be used in research.

\section{Disclosure Statement}

The authors have no conflict of interests to declare.

\section{Funding Sources}

This study was in part supported by New Century Excellent Talents of the Education Department of China and an ISN Research Award from the ISN GO R\&P Committee. The funders had no role in the study design, data collection and analysis, the decision to publish, or the preparation of this paper.

\section{Author Contributions}

Research idea and study design: J. Dong, Y. Zhao, and Y. Zhang. Data acquisition: Y. Zhao, Y. Zhang, Z. Yang, Z. Xiong, J. Liao, L. Hao, G. Liu, Y. Ren, Q. Wang, L. Duan, and Z. Zheng. Statistical analysis: Y. Zhao, Y. Zhang, and J. Dong. Supervision or mentorship: J. Dong. Each author contributed important intellectual content during drafting or revision of this paper and accepts accountability for the overall work by ensuring that questions pertaining to the accuracy or integrity of any portion of this work are appropriately investigated and resolved.

\section{References}

1 Murtagh FE, Addington-Hall J, Higginson IJ. The prevalence of symptoms in end-stage renal disease: a systematic review. Adv Chronic Kidney Dis. 2007 Jan;14(1):82-99.

2 Davison SN, Levin A, Moss AH, Jha V, Brown EA, Brennan F, et al.; Kidney Disease: Improving Global Outcomes. Executive summary of the KDIGO Controversies Conference on Supportive Care in Chronic Kidney Disease: developing a roadmap to improving quality care. Kidney Int. 2015 Sep;88(3):447-59.

3 Scherer JS, Combs SA, Brennan F. Sleep Disorders, Restless Legs Syndrome, and Uremic Pruritus: Diagnosis and Treatment of Common Symptoms in Dialysis Patients. Am J Kidney Dis. 2017 Jan;69(1):117-28.

4 Kraus MA, Fluck RJ, Weinhandl ED, et al. Intensive hemodialysis and health-related quality of life. Am J Kidney Dis. 2016;68: S33-S42.

5 Kang EW, Abdel-Kader K, Yabes J, Glover K, Unruh M. Association of sleep-disordered breathing with cognitive dysfunction in CKD stages 4-5. Am J Kidney Dis. 2012 Dec;60(6):949-58.

6 Williams SW, Tell GS, Zheng B, Shumaker S, Rocco MV, Sevick MA. Correlates of sleep behavior among hemodialysis patients. The kidney outcomes prediction and evaluation (KOPE) study. Am J Nephrol. 2002 Jan-Feb; 22(1):18-28. 
Zhao et al.: Cognitive Decline in Peritoneal Dialysis

7 Griva K, Stygall J, Hankins M, Davenport A, Harrison M, Newman SP. Cognitive impairment and 7-year mortality in dialysis patients. Am J Kidney Dis. 2010 Oct;56(4):693-703.

8 Kalirao P, Pederson S, Foley RN, Kolste A, Tupper D, Zaun D, et al. Cognitive impairment in peritoneal dialysis patients. Am J Kidney Dis. 2011 Apr;57(4):612-20.

9 Smith SC Jr, Jackson R, Pearson TA, Fuster V, Yusuf S, Faergeman O, et al. Principles for national and regional guidelines on cardiovascular disease prevention: a scientific statement from the World Heart and Stroke Forum. Circulation. 2004 Jun;109(25):3112-21.

10 American Psychiatric Association. Diagnostic and statistical manual of mental disorders (DSM-IV). 4th ed. Washington: American Psychiatric Association; 1994.

11 Walters AS, Aldrich MS, Allen R, Ancoli-Israel S, Buchholz D, Chokroverty S, et al.; The International Restless Legs Syndrome Study Group. Toward a better definition of the restless legs syndrome. Mov Disord. 1995 Sep; 10(5):634-42.

12 Walters AS, LeBrocq C, Dhar A, Hening W, Rosen R, Allen RP, et al.; International Restless Legs Syndrome Study Group. Validation of the International Restless Legs Syndrome Study Group rating scale for restless legs syndrome. Sleep Med. 2003 Mar;4(2):121-32.

13 Vignatelli L, Plazzi G, Barbato A, Ferini-Strambi L, Manni R, Pompei F, et al.; GINSEN (Gruppo Italiano Narcolessia Studio Epidemiologico Nazionale. Italian version of the Epworth sleepiness scale: external validity. Neurol Sci. 2003 Feb;23(6):295-300.

14 Lai X, Chen W, Bian X, Wang T, Li J, Wang H, et al. Predictors of poor sleep quality and excessive daytime sleepiness in peritoneal dialysis patients. Ren Fail. 2015 Feb;37(1):61-5.

15 Diagnostic Classification Steering Committee of the American Sleep Disorders Association. International classification of sleep disorders: diagnostic and coding manual. Rochester: American Sleep Disorders Association; 1990.

16 Hatoum HT, Kania CM, Kong SX, Wong JM, Mendelson WB. Prevalence of insomnia: a survey of the enrollees at five managed care organizations. Am J Manag Care. 1998 Jan;4(1):79-86.

17 Merlino G, Piani A, Dolso P, Adorati M, Cancelli I, Valente M, et al. Sleep disorders in patients with end-stage renal disease undergoing dialysis therapy. Nephrol Dial Transplant. 2006 Jan;21(1):184-90.

18 Teng EL, Chui HC. The Modified Mini-Mental State (3MS) examination. J Clin Psychiatry. 1987 Aug;48(8): 314-8.

19 Kurella M, Chertow GM, Luan J, Yaffe K. Cognitive impairment in chronic kidney disease. J Am Geriatr Soc. 2004 Nov;52(11):1863-9.

20 Cheng Y, Wu W, Wang J, Feng W, Wu X, Li C. Reliability and validity of the Repeatable Battery for the Assessment of Neuropsychological Status in community-dwelling elderly. Arch Med Sci. 2011 Oct;7(5):850-7.

21 D’Elia LF, Satz P, Lyons Uchiyama CL, et al. Color Trails Test. Professional manual. Odessa (FL): Psychological Assessment Resources Inc.; 1996.

22 Yeudall LT, Reddon JR, Gill DM, Stefanyk WO. Normative data for the Halstead-Reitan neuropsychological tests stratified by age and sex. J Clin Psychol. 1987 May;43(3):346-67.

23 Randolph C. RBANS manual: Repeatable Battery for the Assessment of Neuropsychological Status. San Antonio: The Psychological Corporation (Harcourt); 1998.

24 Zhang BH, T Y, Zhang WF, et al. The reliability and validity of the Repeatable Battery for the Assessment of Neuropsychological Status. Chin Ment Health J. 2008;22(12):865-9.

25 Yang GG, Tan YL, et al. The application performance of the Repeatable Battery for the Assessment of Neuropsychological Status among normal people in Beijing. Chin Ment Health J. 2010;24(12):926-30.

26 Zung WW. A SELF-RATING DEPRESSION SCALE. Arch Gen Psychiatry. 1965 Jan;12(1):63-70.

27 Zhang YH, Yang ZK, Wang JW, Xiong ZY, Liao JL, Hao L, et al. Cognitive Changes in Peritoneal Dialysis Patients: A Multicenter Prospective Cohort Study. Am J Kidney Dis. 2018 Nov;72(5):691-700.

28 Razeghi E, Sahraian MA, Heidari R, Bagherzadeh M. Association of inflammatory biomarkers with sleep disorders in hemodialysis patients. Acta Neurol Belg. 2012 Mar;112(1):45-9.

29 Sabry AA, Abo-Zenah H, Wafa E, Mahmoud K, El-Dahshan K, Hassan A, et al. Sleep disorders in hemodialysis patients. Saudi J Kidney Dis Transpl. 2010 Mar;21(2):300-5.

30 Zamarian L, Högl B, Delazer M, Hingerl K, Gabelia D, Mitterling T, et al. Subjective deficits of attention, cognition and depression in patients with narcolepsy. Sleep Med. 2015 Jan;16(1):45-51.

31 Kornum BR, Knudsen S, Ollila HM, et al. Narcolepsy. Nat Rev Dis Primers. 2017 3:16100.

32 Huang H, Ghosh P, van den Pol AN. Prefrontal cortex-projecting glutamatergic thalamic paraventricular nucleus-excited by hypocretin: a feedforward circuit that may enhance cognitive arousal. J Neurophysiol. 2006 Mar; 95(3):1656-68.

33 Ooms S, Overeem S, Besse K, Rikkert MO, Verbeek M, Claassen JA. Effect of 1 night of total sleep deprivation on cerebrospinal fluid $\beta$-amyloid 42 in healthy middle-aged men: a randomized clinical trial. JAMA Neurol. 2014 Aug; 71(8):971-7.

34 Chen DW, Wang J, Zhang LL, Wang YJ, Gao CY. Cerebrospinal Fluid Amyloid- $\beta$ Levels are Increased in Patients with Insomnia. J Alzheimers Dis. 2018;61(2):645-51.

35 Olsson M, Ärlig J, Hedner J, Blennow K, Zetterberg H. Sleep deprivation and cerebrospinal fluid biomarkers for Alzheimer's disease. Sleep (Basel). 2018 May;41(5). https://doi.org/10.1093/sleep/zsy025.

36 Madero M, Gul A, Sarnak MJ. Cognitive function in chronic kidney disease. Semin Dial. 2008 Jan-Feb;21(1): 29-37. 
37 Gade K, Blaschke S, Rodenbeck A, Becker A, Anderson-Schmidt H, Cohrs S. Uremic restless legs syndrome (RLS) and sleep quality in patients with end-stage renal disease on hemodialysis: potential role of homocysteine and parathyroid hormone. Kidney Blood Press Res. 2013;37(4-5):458-63.

38 Pengo MF, Ioratti D, Bisogni V, Ravarotto V, Rossi B, Bonfante L, et al. In Patients with Chronic Kidney Disease Short Term Blood Pressure Variability is Associated with the Presence and Severity of Sleep Disorders. Kidney Blood Press Res. 2017;42(5):804-15.

39 Viggiano D, Wagner CA, Blankestijn PJ, Bruchfeld A, Fliser D, Fouque D, et al. Mild cognitive impairment and kidney disease: clinical aspects. Nephrol Dial Transplant. 2019 Apr;gfz051.

40 Bartlett DJ, Rae C, Thompson CH, Byth K, Joffe DA, Enright T, et al. Hippocampal area metabolites relate to severity and cognitive function in obstructive sleep apnea. Sleep Med. 2004 Nov;5(6):593-6.

41 Gunning-Dixon FM, Raz N. The cognitive correlates of white matter abnormalities in normal aging: a quantitative review. Neuropsychology. 2000 Apr;14(2):224-32.

42 Kamba M, Inoue Y, Higami S, Suto Y, Ogawa T, Chen W. Cerebral metabolic impairment in patients with obstructive sleep apnoea: an independent association of obstructive sleep apnoea with white matter change. J Neurol Neurosurg Psychiatry. 2001 Sep;71(3):334-9.

43 Natalia J, Postuma RB, Jacques M, et al. REM sleep behavior disorder and cognitive impairment in Parkinson's disease. Sleep (Basel). 2017;40(8):101.

44 Yarnall A, Rochester L, Burn DJ. The interplay of cholinergic function, attention, and falls in Parkinson's disease. Mov Disord. 2011 Dec;26(14):2496-503.

45 Fulda S, Szesny N, Ising M, Heck A, Grübl A, Lieb R, et al. Further evidence for executive dysfunction in subjects with RLS from a non-clinical sample. Sleep Med. 2011 Dec;12(10):1003-7.

46 Neumann D, Mau W, Wienke A, Girndt M. Peritoneal dialysis is associated with better cognitive function than hemodialysis over a one-year course. Kidney Int. 2018 Feb; 93(2):430-8.

47 Gigli GL, Adorati M, Dolso P, Piani A, Valente M, Brotini S, et al. Restless legs syndrome in end-stage renal disease. Sleep Med. 2004 May;5(3):309-15.

48 Paparrigopoulos T, Theleritis C, Tzavara C, Papadaki A. Sleep disturbance in haemodialysis patients is closely related to depression. Gen Hosp Psychiatry. 2009 Mar-Apr;31(2):175-7. 